PSYCHOMETRIKA-VOL. 32, NO. 2

JणNE, 1967

\title{
THE NUMBER OF WAYS TO LABEL A STRUCTURE*
}

\author{
Frank Harary, Edgar M. Palmer, and Ronald C. Read \\ UNIVERSITY OF MTCHIGAN \\ AND \\ UNIVERSITY OF THE WEST INDIES
}

\begin{abstract}
It has been observed that the number of different ways in which a graph with $p$ points can be labelled is $p$ ! divided by the number of symmetries, and that this holds regardless of the species of structure at hand. In this note, a simple group-theoretic proof is provided.
\end{abstract}

The article by Harary and Read [1966] concluded with a table listing the probabilities $P(n, k)$ that a connected functional digraph with $n$ points has a cycle of length $k$, for $n=2$ to 7 . We wish to acknowledge that the entries in this table are given by the formula

$$
P(n, k)=\frac{(n-1) !}{(n-k) !} \frac{n^{n-k}}{(n-1)^{n}}
$$

in accordance with the theorem in Katz [1955]. This result was anticipated in turn by Rubin and Sitgreaves in an unpublished memorandum cited in Katz [1955].

In order to contribute something positive in this note, we now prove the theorem about graphs and groups which justifies the formula given in Harary and Read [1966] for the number of ways to label a structure. Since this is a sequel to Harary and Read [1966], its notation and terminology will be used. Thus we write $s(G)$ for the symmetry number of graph $G$ (the order of its automorphism group $\Gamma(G)$ ) and $l(G)$ for the number of labelings of $G$. As usual we denote the number of points of $G$ by $p$.

The notation used in the following proof follows that in Harary [in press] and Harary and Palmer [1965]. Accordingly, $S_{p}$ is the symmetric group of degree $p$ acting on $X=\{1,2, \cdots, p\} ; X^{(2)}$ is the set of unordered pairs of the objects in $X ; S_{p}^{(2)}$ is the pair group acting on $X^{(2)}$ as induced by $S_{p}$; and $E_{2}$ is the identity group on $Y=\{0,1\}$. The power group (introduced in Harary and Palmer [1965]) $E_{2}^{S^{(2)}}$ acts on $Y^{X^{(2)}}$ and each function $f$ from $X^{(2)}$ into $Y$ represents a labeled graph with point set $X$. Two points $i, j \varepsilon X$ are considered adjacent in the graph of $f$ whenever $f(\{i, j\})=1$. Health.

*This work was supported by Grant MH 10834 from the National Institute of Mental 
In order to present this proof concisely, we assume the basic properties of permutation groups $A$ acting on $X$. These include the "stabilizer" of an object $x \varepsilon X$ (the subgroup of $A$ which fixes $x$ ), the "orbit" of $A$ which contains $x$ (the set of all objects to which $x$ can be mapped by permutations in $A$ ), and the "index" of a subgroup $B$ of $A$ (the ratio of the order of $A$ to that of $B$ ). We also recall the well known result:

Lemma. The index in the group $A$ of the stabilizer $A_{x}$ of an object $x \varepsilon X$ is the number of objects in the orbit of $A$ which contains $x$.

The theorem is stated for graphs, but is easily modified to apply to any type of structure, e.g., trees, directed graphs, tournaments, relations, 1-choice structures (functional digraphs), and nets.

Theorem. The number of different ways in which the points of $G$ can be labeled is:

$$
l(G)=\frac{p !}{s(G)} .
$$

Proof. Since the theorem is obvious for $p=1,2$, we assume $p \geq 3$.

Now let $G$ be the unlabeled graph on $p$ points which corresponds to the function $f$ mentioned above. It is clear that the number of ways in which $G$ can be labeled is simply the number of functions in the orbit of $f$ regarded as an element in the object set of the power group $E_{2}^{S_{p}(2)}$. Furthermore, the stabilizer of $f$ in $E_{2}^{S_{p}(a)}$ is obviously isomorphic to $\Gamma(G)$. Applying the lemma to this power group, we have the result that the number of ways of labeling $G$ is the order of $E_{2}^{S_{\beta_{(2)}}}$ divided by the order of $\Gamma(G)$, i.e., the index of $\Gamma(G)$ regarded as a subgroup of the power group. The proof is completed by observing that the order of this power group is $p$ ! when $p \geq 3$.

\section{REFERENCES}

Harary, F. A seminar on graph theory. New York: Holt, Rinehart and Winston, 1967. Harary, F. and Palmer, E. M. The power group of two permutation groups. Proceedings of the National Academy of Science, U.S. A., 1965, 54, 680-682.

Harary, F. and Read, R. The probability of a given 1-choice structure. Psychometrika, 1966, 31, 271-278.

Katz, L. Probability of indecomposability of a random mapping function. Annals of Mathematical Statistics, 1955, 26, 512-517.

Manuscript received $\% / 25 / 66$ 